\section{Sicherheitsfibel Chemie 1972}

Obgleich keine Chromatographie-Information, sei hier trotzdem auf diese äußerst nützliche Publikation hingewiesen:

Sicherheitsfibel Chemie, 1972, von Roth, 160 Seiten. Verfasser: Dr. med. H. Roth und Dr. rer. nat. L. Roth, D-75 Karlsruhe, Devrientstraße 6.

Druck: C. F. Müller, Großdruckerei und Verlag,

D-75 Karlsruhe, Rheinstraße 122.

Preis: DM 18,-

Die Fibel enthält unter anderem:

Gefahrenbezeichnungen für gefährliche chemische Stoffe (R-Hinweise)

Anschriftenverzeichnis für Notfälle

Verzeichnis der Stoffe (rund 380 Stoffe und 65 Stoffgruppen. Hiervon sind 68 Stoffe und 28 Stoffgruppen, die besonders häufig verwendet werden, ausführlich beschrieben)

MAK-Werte, Dampfdruck, Flammpunkte (1972)

Zubereitung, Kennzeichnung der Zubereitung, Einstufung, Verpackung und Kennzeichnung von Zubereitungen gefährlicher Stoffe

Verordnungen (z.B. über brennbare Stoffe, Giftverordnung der Länder)

Ausrüstungsvorschläge für die Arbeitssicherheit in Betrieben und Laboratorien wie Atemschutz, Feuerschutz, Augenschutz, Raumschutz, Schutzkleidung, Verbandzeug, Arzneimittel, Reinigungsmittel, Adsorptionsmittel, Kennzeichnung, Warnsymbole, Literatur.

Sicherheitsratschläge für gefährliche chemische Stoffe Länder-Giftinformationszentren (Deutschland, Schweiz, Niederlande, Österreich) mit Adressen, Telefon und solchen Angaben, wann erreichbar und wer zuständig in der Durchwahl bzw. was zu tun, wenn besetzt ist.
Die stoffliche Fülle ist in einer brauchbaren signalisierten Unterteilung aufbereitet:

$\mathrm{N}=$ Name (Namen) in deutsch und seine Synonyma, in englisch, französisch, niederländisch, italienisch, mit Nummernangabe, welche zum Ort in der Sammlung der Unfallverhütungsvorschriften führt

$\mathbf{P}=$ Physikalische Daten (Siedepunkt, Schmelzpunkt, spezifisches Gewicht des Stoffes und spezifisches Gewicht seiner Gase bzw. Dämpfe, sowie die Löslichkeit in Wasser)

F oder $\mathbf{R}=$ Feuerbekämpfungsdaten oder besondere Gefahren, Risiken

$\mathrm{E}=$ Erscheinungsbild und häufige Verwendungsarten, in einigen Fällen auch der Geruchsschwellenwert

$\mathrm{U}=$ Umgang

$G=$ Gesundheitsgefährdung

$\mathrm{S}=$ Sicherheits- und Schutzmaßnahmen

$\mathbf{H}=$ Hilfsmaßnahmen, und zwar ausschließlich Hinweise für die erste Hilfe von Laien, aber auch Literaturhin. weise für den Arzt unter Stichworten

$\mathrm{V}=$ Versandvorschriften

$\mathrm{K}=$ Kennzeichnungsvorschrift

$\mathrm{L}=$ Literatur, die wichtigsten Literaturstellen für jeden Stoff

Der Rezensent hat in nicht wenigen Meßlaboratorien, in denen vorwiegend meßtechnisch und physikalisch ausgebildetes Personal tätig ist, chemische Kenntnisse über Stoff. gefahren vermißt. Zwar verwendet der Analy tiker oft nur $10^{-3} \mathrm{~g}$ der Proben, aber die eingehenden Probenmengen liegen häufig im Falle gefährlicher Stoffe über der tödlichen Dosis, wobei besonders Anreicherungen aus der präparativen Chromatographie höchst gefährlich sein kön. nen. Diese Sicherheitsfibel ist daher auch für die chromato. graphisch tätigen Analytiker von ganz besonderem Wert. Ihr Studium und ihre rasche Zugreifbarkeit an jedem Arbeitsplatz wird dringend empfohlen.

Rudolf E. Kaiser 